\title{
Erratum to: Risk-Sensitive Control with Near Monotone Cost
}

\author{
Anup Biswas • V.S. Borkar • K. Suresh Kumar
}

Published online: 13 April 2010

(C) Springer Science+Business Media, LLC 2010

\section{Erratum to: Appl Math Optim DOI 10.1007/s00245-009-9096-7}

We have used Theorem 3 of [2] in the proof of Theorem 2.1 in order to claim $\rho \leq \beta$, and also in a remark preceding Theorem 2.2. The proof thereof in [2], however, is flawed. Our own proof of the reverse inequality $\rho \geq \beta$ also has gaps. We give an alternative proof of $\rho=\beta$ below that sidesteps both difficulties.

Let $\chi_{n}$ denote a nonnegative smooth function such that $\chi_{k} \equiv 1$ in $B_{k}:=\{x:\|x\| \leq$ $k\}, \chi_{k} \equiv 0$ in $B_{k+1}^{c}$ and $0 \leq \chi_{k} \leq 1$. Let $r_{k}=r \chi_{k}$. Define for $\alpha>0$,

$$
u_{k}^{\alpha}(\theta, x):=\inf _{v \in M_{1}} E_{x}\left[e^{\theta \int_{0}^{\infty} e^{-\alpha t} r_{k}\left(X_{t}, v_{t}\right) d t}\right]
$$

The online version of the original article can be found under doi:10.1007/s00245-009-9096-7.

\footnotetext{
A. Biswas

Tata Institute of Fundamental Research, Centre for Applicable Mathematics, Bangalore 560065, India

e-mail: anup@math.tifrbng.res.in

V.S. Borkar $(\bowtie)$

School of Technology and Computer Science, Tata Institute of Fundamental Research, Mumbai 400005, India

e-mail: borkar@tifr.res.in

K. Suresh Kumar

Department of Mathematics, Indian Institute of Technology Bombay, Mumbai 400076, India e-mail: suresh@math.iitb.ac.in
} 
Let $\tau^{v}$ denote the entrance time to the ball $B_{k+1}$ under the Markov control $v \in M_{1}$. Let $x \in B_{k+1}^{c}$. Then standard dynamic programming argument gives

$$
u_{k}^{\alpha}(\theta, x) \leq \inf _{M_{1}} E_{x}\left[u_{k}^{\alpha}\left(\theta, X_{\tau^{v}}\right)\right] \leq \sup _{y \in \partial B_{k+1}} u_{k}^{\alpha}(\theta, y)
$$

Here we used the facts that $r_{k} \equiv 0$ on $B_{k+1}^{c}$ and $e^{-\alpha \tau^{v}}<1$ a.e. By closely mimicking the arguments of Theorem 2.1, one has

$$
\log u_{k}^{\alpha}(\theta, x)-\log u_{k}^{\alpha}(\theta, 0) \rightarrow \phi^{k}(\theta, x)
$$

in $W_{l o c}^{1, p}\left(\mathbb{R}^{n}\right), p \geq 2$, along a subsequence. By the Sobolev embedding theorem, the convergence is uniform on compact subsets of $\mathbb{R}^{n}$. But the foregoing arguments show that for $x \in B_{k+1}^{c}$,

$$
\begin{aligned}
& \log u_{k}^{\alpha}(\theta, x)-\log u_{k}^{\alpha}(\theta, 0) \leq \sup _{y \in \partial B_{k+1}}\left[\log u_{k}^{\alpha}(\theta, y)-\log u_{k}^{\alpha}(\theta, 0)\right] \\
& \Rightarrow \phi^{k}(\theta, x) \leq K(k),
\end{aligned}
$$

where $K(k)$ can be chosen independent of $x \in \mathbb{R}^{n}$. Set $\hat{u}^{k}=e^{\theta \phi^{k}}$. We set $\theta=1$ without loss of generality and suppress the $\theta$-dependence now on for notational simplicity. Using arguments as in the proof Theorem 2.1 , we have $\left(\hat{u}^{k}, \rho_{k}\right)$ satisfying

$$
\rho_{k} \hat{u}^{k}=\inf _{v \in V_{1}}\left[L \hat{u}^{k}(x, v)+r_{k}(x, v) \hat{u}^{k}\right]
$$

with $\hat{u}^{k}(0)=1$. Moreover, $\hat{u}^{k}$ is bounded above by $e^{K(k)}$. This fact allows us to show, using a standard argument based on Ito's formula, that

$$
\rho_{k} \leq \inf \limsup _{T \rightarrow \infty} \frac{1}{T} \log E\left[e^{\int_{0}^{T}\left(r_{k}\left(X_{t}, v_{t}\right)\right) d t}\right],
$$

where the infimum is over all admissible controls. Since $\left|r_{k}\right| \leq|r|, \rho_{k}$ 's are uniformly bounded in $k$. Since $\hat{u}^{k}(0)=1$ and the coefficients of (1) are bounded, we have $\hat{u}^{k}$ uniformly bounded on compact sets by Harnack's inequality (p. 199, [1]). Thus using Theorem 9.11, page 235, [1], we have $\hat{u}_{k} \rightarrow \hat{u}$ in $W_{l o c}^{1, p}\left(\mathbb{R}^{n}\right)$ and $\rho_{k} \rightarrow \rho$ along a subsequence. Furthermore, it follows from Harnack's inequality that $\hat{u}>0$, in fact one has uniform positive lower bounds on $\hat{u}^{k}$ on compacts. Letting $k \rightarrow \infty$ in (1), $(\rho, \hat{u})$ satisfies

$$
\rho \hat{u}=\inf _{v \in V_{1}}[L \hat{u}(x, v)+r(x, v) \hat{u}] .
$$

For any admissible control $v_{t}$, (2) yields

$$
\limsup _{T \rightarrow \infty} \frac{1}{T} E_{x}\left[e^{\int_{0}^{T} r_{k}\left(X_{t}, v_{t}\right) d t}\right] \geq \rho_{k} .
$$

Therefore using the fact that $r_{k} \leq r$ and letting $k \rightarrow \infty$ we have

$$
\limsup _{T \rightarrow \infty} \frac{1}{T} E_{x}\left[e^{\int_{0}^{T} r\left(X_{t}, v_{t}\right) d t}\right] \geq \rho .
$$


Hence $\beta \geq \rho$.

To establish the reverse inequality, let $\hat{u}$ and $\rho$ be as above and $v^{*}$ be a minimizing measurable selector in (3). By the near monotonicity assumption, $\inf _{v} r(\cdot, v)>\beta \geq \rho$ outside a compact set $C$. We know that for some $v>0, \hat{u} \geq v>0$ in $C$. Let $x \in C^{c}$. Let $\tau_{C}$ denote the hitting time to $C$. By Fatou's lemma,

$$
\hat{u}(x) \geq E_{x}\left[e^{\int_{0}^{\tau} C}\left(r\left(X_{t}, v_{t}^{*}\right)-\rho\right) d t \hat{u}\left(X_{\tau_{C}}\right)\right] \geq v .
$$

This proves that $\hat{u}$ is bounded below by $v$. Using Ito's formula and Fatou's lemma, we have

$$
\hat{u}(x) \geq E_{x}\left[e^{\int_{0}^{T}\left(r\left(X_{t}, v_{t}^{*}\right)-\rho\right) d t} \hat{u}\left(X_{T}\right)\right] \geq v E_{x}\left[e^{\int_{0}^{T}\left(r\left(X_{t}, v_{t}^{*}\right)-\rho\right) d t}\right] .
$$

Taking log, dividing by $T$ and letting $T \uparrow \infty$ on both sides, we have

$$
\limsup _{T \rightarrow \infty} \frac{1}{T} E_{x}\left[e^{\int_{0}^{T} r\left(X_{t}, v_{t}^{*}\right) d t}\right] \leq \rho .
$$

Thus $\beta \leq \rho$. Moreover it follows from (4) and (5) that $v^{*}$ is optimal for the risk sensitive control problem. This completes the proof of Theorem 2.2.

\section{References}

1. Gilbarg, G., Trudinger, N.S.: Elliptic Partial Differential Equations of Second Order, 2nd edn. Springer, Berlin (2001)

2. Runolfsson, T.: Risk-sensitive control of stochastic hybrid system on infinite time horizon. Math. Probl. Eng. 5, 459-478 (2000) 